\title{
Paste backfill reticulation optimisation using high shear mixing at DeGrussa Mine
}

\author{
NR Steward Weir Minerals Australia, Australia \\ G Allen Sandfire Resources, Australia \\ K Tiedermann Sandfire Resources, Australia
}

\begin{abstract}
This paper investigates the work carried out by DeGrussa Mine and the Weir Technical Centre (WTC) of Weir Minerals Australia, in developing a process to produce a consistent cemented paste backfill that is fully sheared and presents the lowest possible yield stress and pressure gradients during the underground transport phase.

The existing twin shaft paste mixer on DeGrussa Mine was not providing sufficient shear or time of shear to deliver a fully homogenised product. The project to improve the paste backfill rheology involved quantifying the performance of the twin shaft mixer as well as the required rheological parameters of yield stress and pressure gradient of the paste backfill product. In order to achieve the required outcome, a centrifugal pump was installed after the mixer to provide the shear energy required to produce a fully sheared paste backfill rapidly and continuously.

A fully sheared consistent paste backfill is required to ensure predictable transport of the paste backfill throughout the DeGrussa Mine reticulation system. This predictability of paste backfill performance results in a safe and robust reticulation system, together with ensuring pipeline integrity. The lower pressure gradients, manifest by the fully sheared paste backfill, also allow DeGrussa Mine to fill stopes that are at a distance that would otherwise require a positive displacement pumped system.
\end{abstract}

Keywords: paste, backfill, pressure gradient, shear, mixing, rheometry, yield stress, slump, centrifugal pump

\section{$1 \quad$ Background}

Paste backfill distribution systems around the world are plagued by problems typically associated with the build-up of tailings within the pipeline. The worst-case scenario is blockage and bursting of the pipeline while the most benign cases result in reductions in flow rates, or higher operating pressures.

The fact that these are not uncommon events is demonstrated by paste backfill reticulation systems providing for tee pieces that have caps that can be blasted off, dump valves at the bottom of vertical drops and inline tee pieces with dump valves to drain blocked systems.

It is not uncommon for mining operations to have an operating backfill pipeline that is half full of settled, and in some cases cemented backfill (Figure 1). 

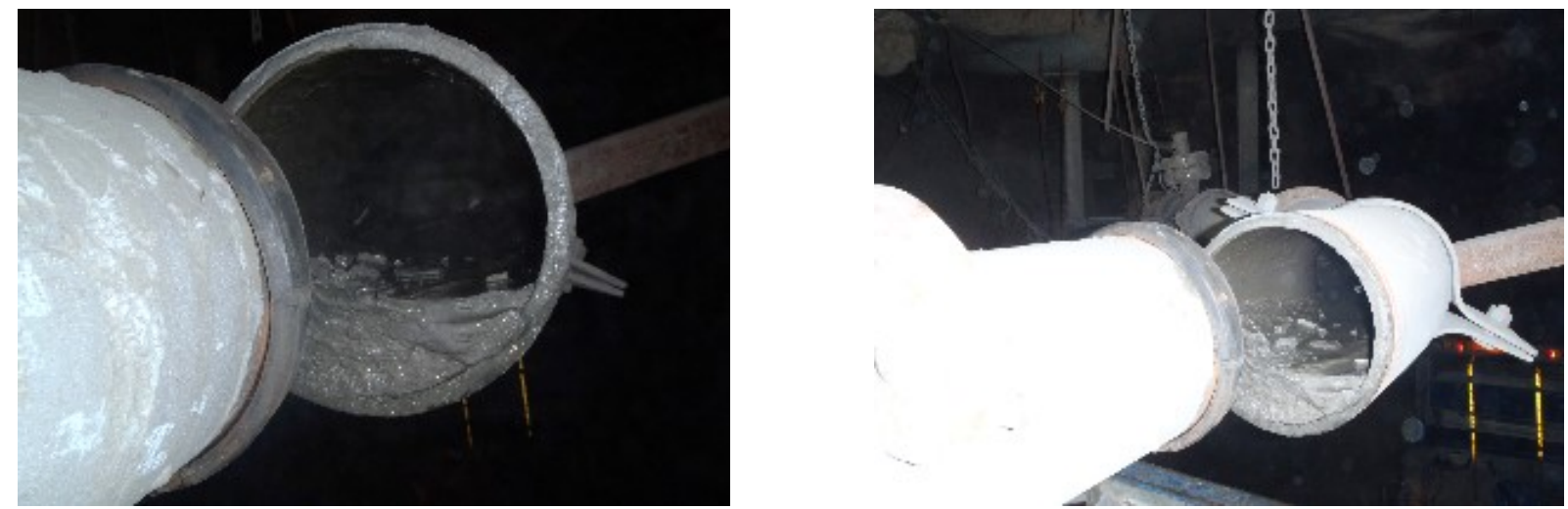

Figure 1 Partially filled paste backfill pipeline

These problems are typically functions of poor backfill system design and operation.

The design of a backfill reticulation system is based on several factors associated with the backfill itself and the physical reticulation system layout:

- Solids concentration.

- Solids particle size distribution (PSD).

- Mineralogy.

- Physico-chemical implications.

- Volumetric flow rate.

- Reticulation route and characteristics.

- Backfill homogeneity.

All these factors affect the pipeline friction losses which are the fundamental design building blocks of any pipeline reticulation system. The friction loss, or pressure gradient, is the loss in pressure owing to the friction of the backfill with the pipe wall and its interaction through the paste. It is simplistically the pressure required to push the backfill $1 \mathrm{~m}$ at the required flow rate, or pipeline velocity, in a specific pipe size and is typically reported in $\mathrm{kPa} / \mathrm{m}$.

The pressure gradient together with the paste backfill reticulation route, changes in elevation, fittings losses and any further pressure exerted on the system, such as that owing to pumping, are the factors used to develop a safe and robust reticulation system.

\section{Backfill reticulation systems}

The initial design of backfill distribution systems involved both 'full flow' and 'free fall' dedicated systems shown in Figures 2 and 3 respectively.

Full flow systems are typically the design objective in backfilling operations today. A full flow system is one in which the system pressure losses owing to friction with the pipe wall and fittings together with any entrance and exit losses balanced by the gravity head due to the difference in elevation between the discharge point and the delivery point on surface.

For such systems, no air-backfill interface exists and the backfill fills the pipeline system from the surface to the exit at the stope being filled.

The advantages of a full flow system are:

- Increased pipe lifetime owing to reduced wear, therefore reduced downtime for pipe repairs.

- A controlled system that can be monitored for any anomalies improving safety. 


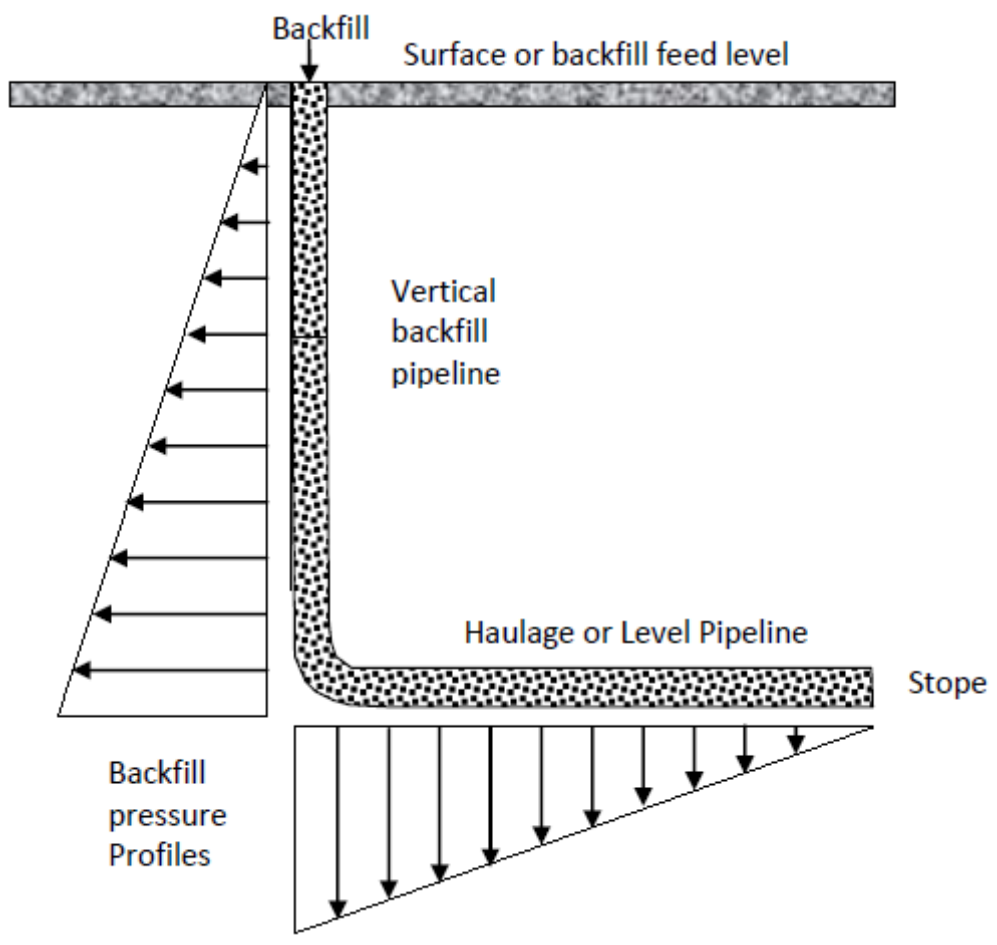

Figure 2 Full flow

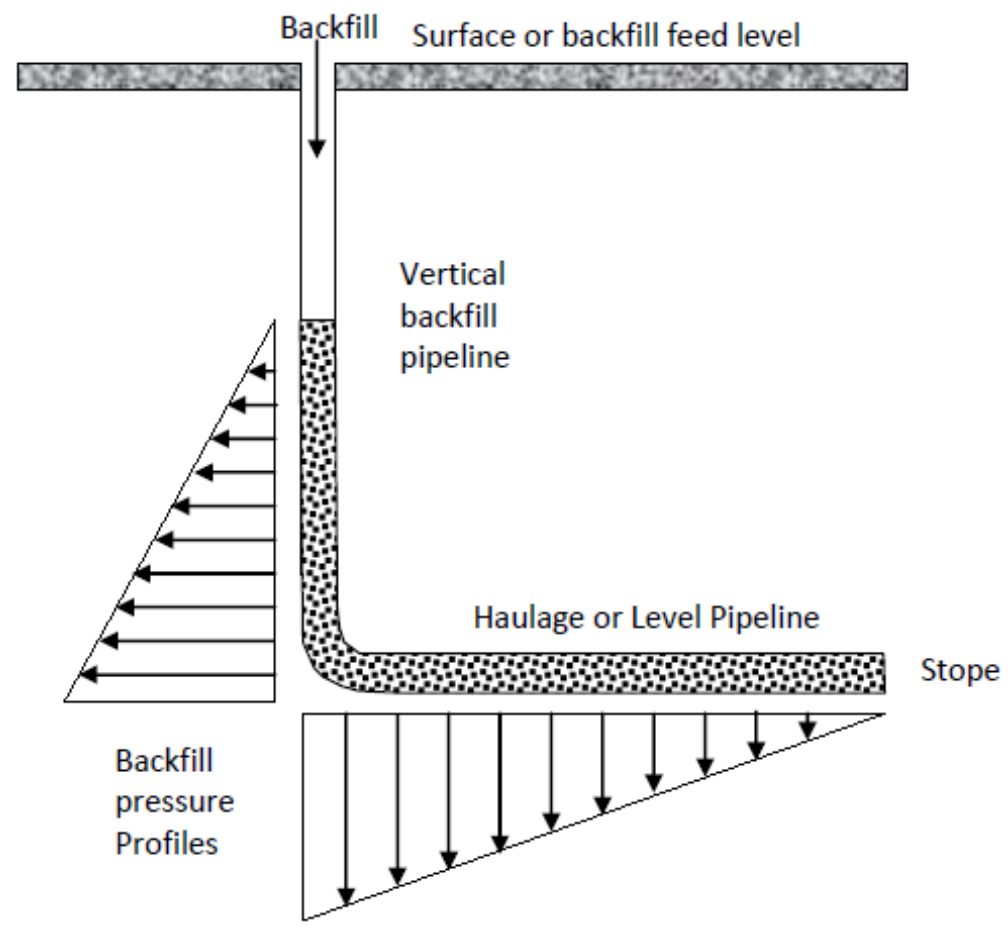

Figure 3 Free fall

The alternative, which is typically the standard in Australia, is the free fall system.

Free fall pertains to a transport condition existing in any vertical column in the backfill reticulation system, where the solids fall in air to a backfill/free fall zone interface. The position of this interface, in the vertical column, and the pressure head that it develops due to its height is dependent on the pipeline pressure gradients due to the flow rate of the specific backfill product within the pipe. Thus, if there is an increase in the backfill flow rate there is an increase in the pipe pressure gradients resulting in an increased interface 
height to provide the increase in head necessary to drive the backfill to the stope under the new flow conditions.

It is this 'self-regulating' nature of the freefall system that has suggested that accurate predictions of pipeline pressure gradients are not required. If any changes are made to the either the backfill mix design, characteristics, or the flow rate, the interface height will change until the required pressure head to drive the backfill, at the required flow rate, to the stope is achieved. In designing these systems in the past, the predictions of pressure gradients were conservatively high in order to ensure the safe pressure rating of pipes.

The delivery of backfill from the surface storage to the pipeline is through a flooded surge cone, sometimes with a restriction orifice in the pipeline to regulate the flow rate. The main advantage of the freefall system of backfilling was the belief that high operating pressures could be avoided in the haulage and shaft piping. If, however the pipeline line blocked underground it would fill to the surface with backfill resulting in the full static head being experienced by the underground pipeline anyway. The disadvantages of freefall are numerous and over time, tend to result in catastrophic failure:

- Inlet static pressures are below atmospheric and therefore air is sucked in through pipe joints and pipe linings can be pulled off.

- Excessive pipe wear due to the high velocities of the particles in freefall (Figure 4).

- Pipe bursting failure due to cyclic impact loading at the air-backfill interface. While this may be considered irrelevant in a borehole environment there is sufficient proof of the country rock being spalled at the air/backfill interface, once the steel lining is worn through, if a steel sleeve is used, resulting in the spalled country rock falling into the borehole causing a blockage.

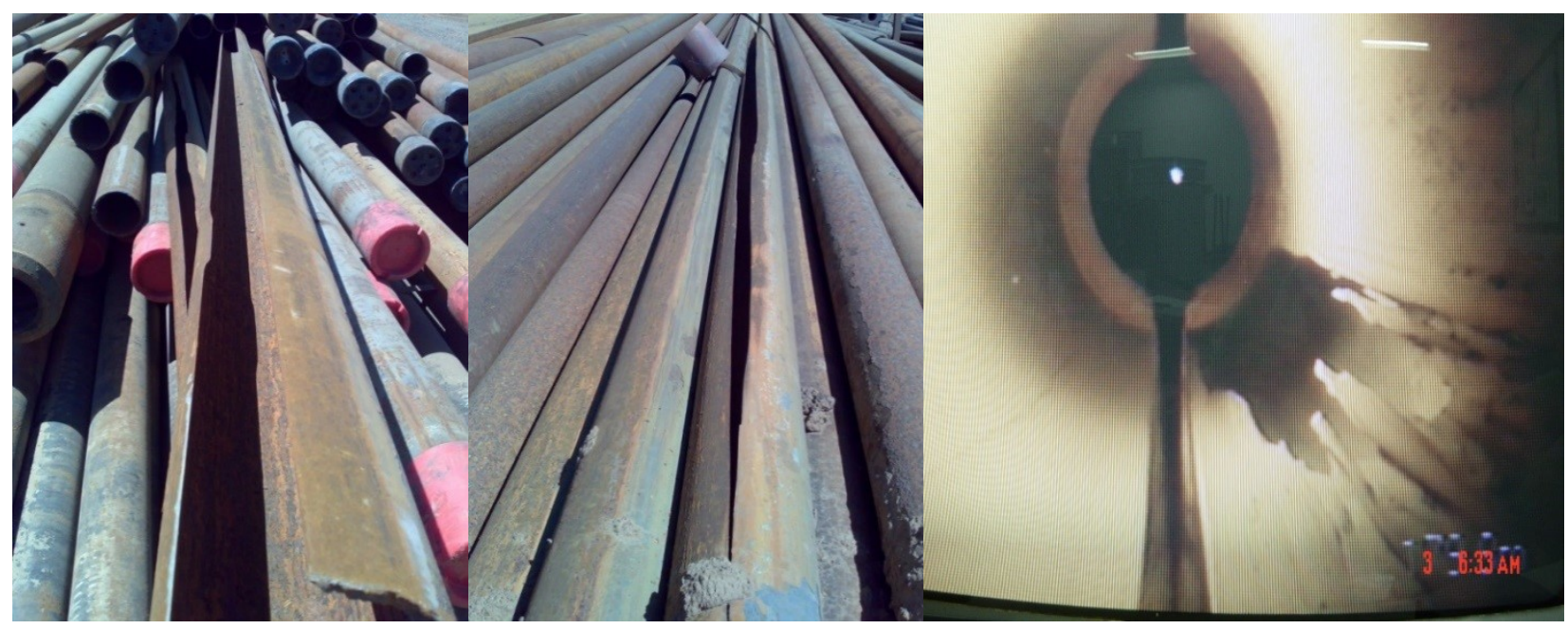

Figure 4 Typical pipe freefall failures and a camera logging showing the 'freefall' striation wear in situ

Excessive pipe wear and blockages are a fact of paste backfilling in Australia. It is not necessarily the pipeline designers' intention to design a free fall system, it is quite possibly the incorrect determination of pipeline pressure gradient that is responsible for the incorrect selection of pipe size. This, together with inconsistent, or incomplete, paste backfill manufacture, which exacerbates the incorrect rheological characterisation of the paste backfill, results in the free fall typical in Australian backfill systems. 


\section{Pressure gradient determination}

The measurement of pressure gradients is typically carried out by one of the following methods (Figure 5):

- Laboratory scale rotational viscometry.

- Closed loop pipeline test work.

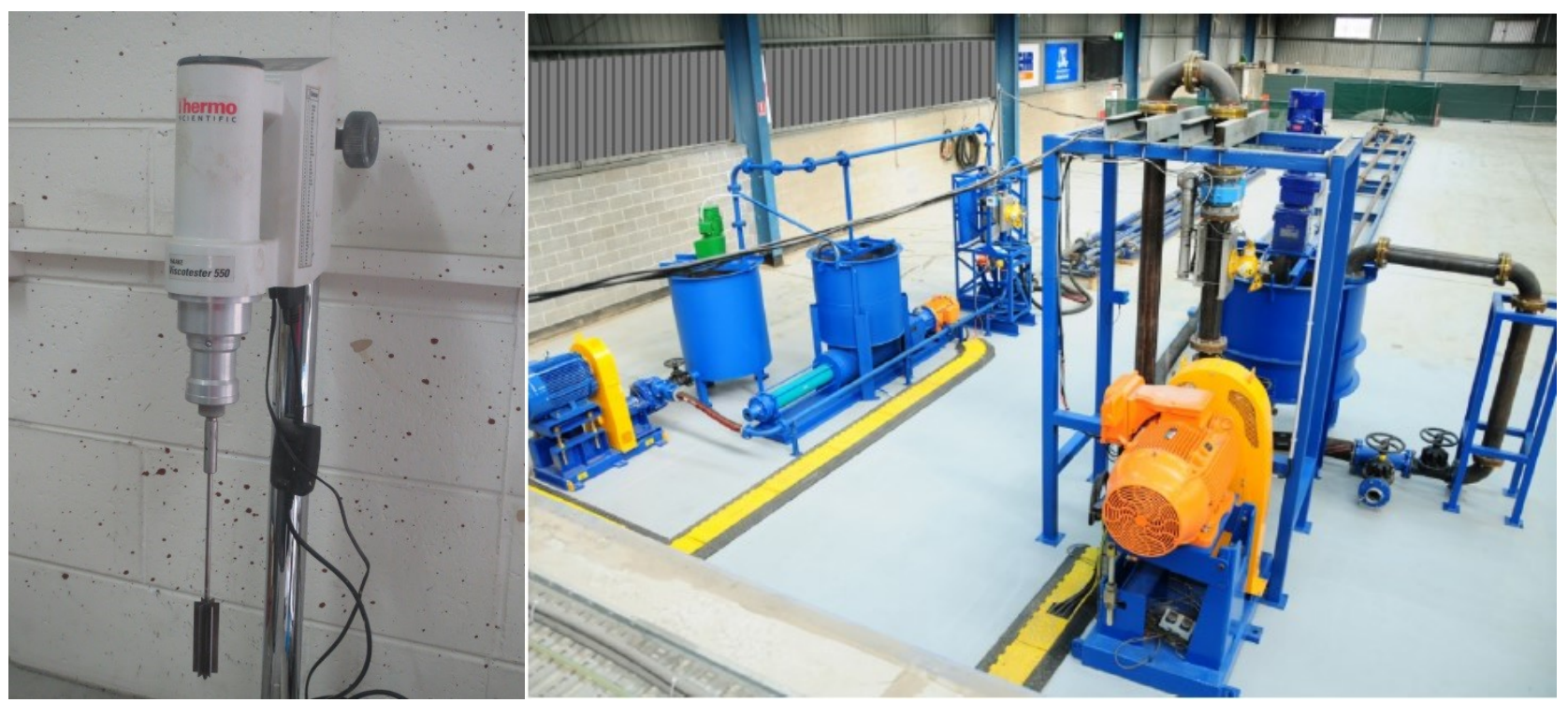

Figure 5 Laboratory rheometer and the Weir Technical Centre pipe loop facility

There are significant differences in the two test methods; sample size, test methodology and most importantly, the outcomes. The Weir Technical Centre (WTC) carries out pipe loop test work regularly for pipeline system designs and simultaneously carries out laboratory scale rheometry. The WTC has not achieved a constant relationship between the two test methods.

Further bench scale type tests are used to determine the 'rheology' of paste backfills such as various slump techniques. These test methods have also deviated from pipe loop test data.

The pressure gradient, besides being incorrectly determined by bench scale measurements when compared to pipe loop test data, is further misrepresented owing to the typical paste backfill manufacturing methods.

\section{$4 \quad$ The DeGrussa Mine backfill system investigation}

\subsection{The paste backfill system overview}

The DeGrussa Mine, of Sandfire Resources, has addressed the issue of both pressure gradient determination and site operational aspects of paste backfill manufacture to optimise their reticulation system. The issues associated with both pipeline reticulation and the manufacture of the paste backfill, together with the resulting solutions, are presented here.

The DeGrussa Mine paste backfill production system comprises primarily:

- A cycloning system to separate the flotation tailings into a coarse and fine fraction.

- A thickener to increase the solids concentration of the cyclone overflow for delivery to the tailings storage facility.

- A vacuum filter belt to dewater the cyclone underflow.

- A twin shaft mixer to recombine the dewatered cyclone underflow, a bleed stream of unfiltered cyclone underflow for density adjustment, and a hydraulic binder. 
The cycloning of the flotation tailings improves the efficiency of the vacuum filter belt to dewater the cyclone underflow through only dealing with the coarse tailings.

The total DeGrussa Mine paste backfill system was audited during the test and development program which included a review of the quality control processes and procedures. The quality control processes included:

- Sampling the filter cake for moisture content measurement.

- Sampling the final paste backfill for:

○ Viscosity.

- Slump.

- Core manufacture.

- Testing of the cured cores for:

$\circ$ Strength.

○ Sulphide content.

The audit process resulted in recommendations, to improve the processes for testing samples, which were implemented by the mine resulting in a more representative and consistent set of test results.

\subsection{Paste backfill reticulation optimisation using shear mixing at De Grussa Mine}

During the audit a clear issue associated with mixing of the paste backfill was observed. The final product from the paste mixer indicated significantly lower slump and higher yield stress when compared to the final product discharged into the stope underground. The yield stress value of the paste backfill could be in the order of $200 \%$ lower underground compared to the surface. This indicated further reduction in viscosity or product rheology due to mixing and shearing of the material, which was occurring throughout the pipeline system.

This has a significant effect on the paste backfill system operation where a 'high viscosity' paste backfill manifests higher pressure gradients in the system, such that when an increase in the pressure gradient to a specific level is experienced, water is added to the system to improve flowability. This action affects the quality and performance of the final placed product as regards to strength and stope support and stability, i.e. the addition of water increases the water binder ratio and decreases the final paste backfill strength.

The pressure gradient on DeGrussa Mine is continuously monitored as the difference in pressure between two pressure transducers underground on a level section of piping.

This problem of paste backfill flowability has an effect on the mining operation as backfilling of the farthest stopes cannot be achieved with the higher viscosity paste backfill without either; running at a lower paste backfill solids concentration than the strength design requires, or using a positive displacement piston pump.

The options as to why there is an increase in pressure gradient are:

- Increase in the fines content.

- Incorrect dosage of binder.

- Increased solids concentration.

The PSD of the tailings, while variable over time maintains a consistent envelope of size range. The cycloning methodology has, in the past, resulted in a variable final cyclone underflow PSD; variation was not sufficiently regular to match the variation in pressure gradients seen during underground operations. Similarly, the addition of binder did not follow the consistent variation in pressure gradient. 
Investigations indicated that the moisture content of the filter cake on the conveyor varied little and moisture content modification, through the addition of unfiltered cyclone underflow slurry, could be made. Moisture content control in the paste mixer was based on routine sample collection of the filter cake for density and yield stress measurement, and as an interim inferred value, the mixer power draw. Using power draw as a method of control to determine paste backfill viscosity is conducted at other paste backfill operations worldwide. This, together with the actual final solids concentrations measured suggests that the solids concentration of the final paste backfill remains consistent.

What had been established in Canada in the 1990s was a difference in slump measurements on the surface to those underground at the stope. Further test work by Bouchard-Hébert Mine in the 1990s indicated that an extended mixing of the paste backfill resulted in a decreasing viscosity. This has over the years been attributed, erroneously, to shear thinning. It should be considered as 'thinning' with time of shear.

Shear thinning is a rheological term indicating that the rate of wall shear stress increase decreases with increasing shear rate, or in a pipeline, flow rate. Simplistically as the slurry flow rate through a pipe increases the rate of pressure gradient increase, decreases. This is a reversible state: decrease the shear, or flow rate, and the wall shear stress returns to the same level at the same rate of shear, or flow rate in a pipe.

In terms of viscosity, shear thinning owing to an increased shear rate results in a decrease in the viscosity of the paste backfill, as the rate of shear returns, decreases, to its original starting point the viscosity increases back to the initial viscosity as well.

This is different to the non-reversibility of the yield stress decrease and flowability increase existing when a typical paste backfill is mixed for extended periods of time.

This thinning with time of shear is a clear indication that the paste backfill is poorly or insufficiently mixed in the paste backfill mixer.

The process of vacuum filtration, using belts or discs, results in the particles being brought into contact with each other and the water that is not drained from the system being forced into the interstitial cavities between the particles. The retained moisture content can vary between 15 and $25 \%$. The styled 'dry' tailings or 'filter cake' is a product that has been mechanically dewatered to produce a product held together by the mechanical interlocking of the particles through packing and particle-particle friction. The cake can be handled mechanically immediately after manufacture owing to its mechanically interlocked state. In this state the product appears to be dry.

While the 'dry' tailings do not bleed water; they do contain interstitial water. The amount of interstitial water is dependent on the PSD and shape.

The curve in Figure 6 indicates the nature of the change in the porosity of a slurry as the water is lost.

The state of the water in the solids can be explained as:

- Surface adsorbed - water is adsorbed in the particle's surface granularity.

- Contact point - as more water is added it concentrates at particle contact points forming water bridges and binding the grains together owing to water tension. This results in sample bulking owing to the water tension hindering particle rearrangement.

- Capillary water - as more water is added this displaces air and increases the water tension capability. This capillary tension increases to a maximum as the degree of water saturation approaches $100 \%$. At the optimum water/solids ratio where the capillary water tension is greatest the minimum porosity is attained, i.e. when the particles are pulled together with the greatest water tension.

- Slurry - at a maximum capillary tension the addition of further water reduces this tension and effectively dilutes the suspension and increases the porosity, by separating particles no longer held by water tension, as the water content increases up the saturation line. 


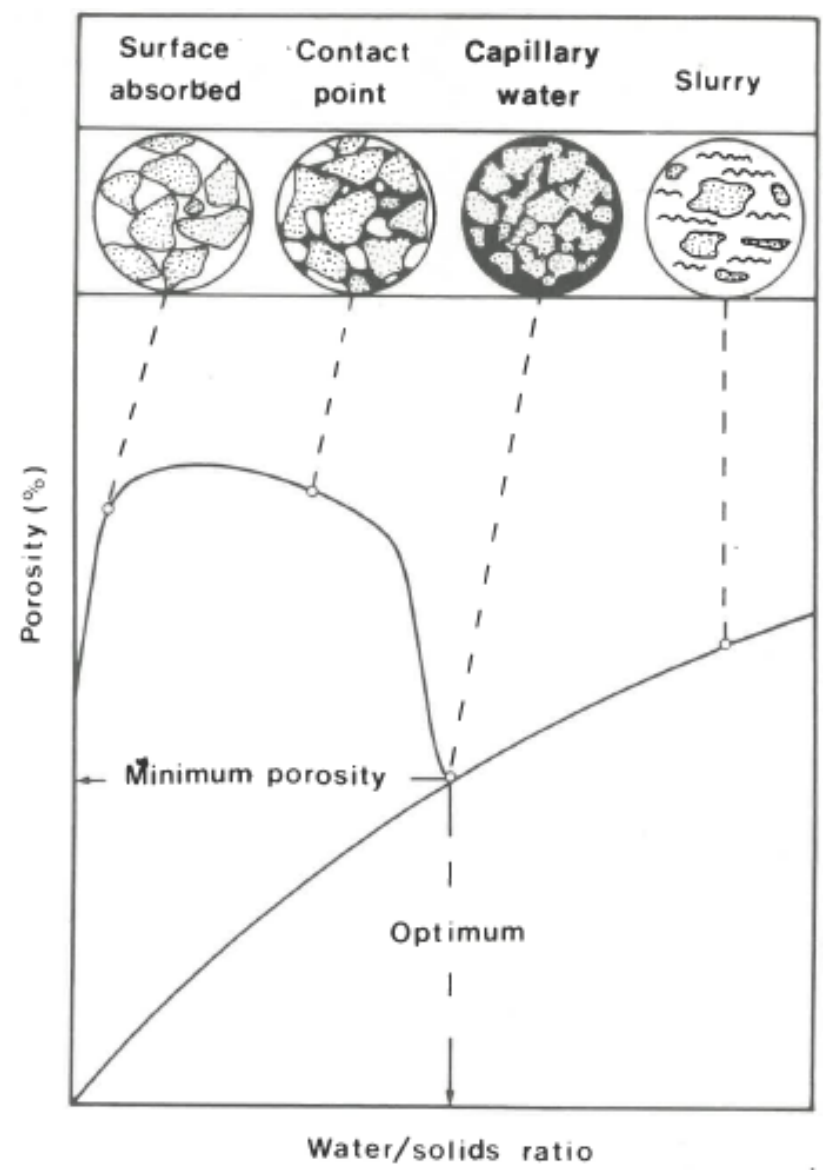

Figure 6 Placement properties curve indicating the nature of water in backfill (after Clark 1988)

In order to have a consistent homogeneous paste backfill it is necessary to both break up any mechanical interlocking and distribute the interstitial water, and any further water added, evenly over and between every particle present.

This requires sufficient energy to be transferred to the filter-cake by a mixing system. This is not typically achieved in paste backfill systems as indicated by the changing rheology with pipeline transport or further mixing. The immediate result is that the retained mechanical interlocking results in a far higher yield stress measurement or slump and interpretively higher-pressure gradients. This results in larger pipe sizes being specified than a fully mixed product would require, i.e. larger pipe sizes are used to decrease the pressure gradient for a specific flow rate.

The outcome of this action is that as the paste backfill undergoes further mixing through shear in the pipeline, decreasing the pressure gradient, increasing the flow rate resulting in freefall in the vertical sections of pipeline. Freefall results in high pipeline velocities, wear and the failures associated with paste backfilling.

Thus, a well-designed paste reticulation system is based on a fully sheared homogenised paste backfill product and a mixing system to achieve this.

DeGrussa Mine approached this problem by first identifying the magnitude of the yield stress variation associated with samples taken at the mixer and exposed to further shear. This involved taking samples of the cemented paste mixer product at $78 \mathrm{wt} \%$ solids and subjecting it to further shear in an industrial bowl mixer. After each mixing time increment the paste backfill was tested again for yield stress using a laboratory bench rheometer as well as slump using a $100 \mathrm{~mm}$ diameter slump cylinder. A further test was carried out underground with a sample of the paste backfill before it entered the stope. The cylinder slump heights have been converted to a yield stress using the Pashias et al. (1996) relationship. The results of this test work are provided in Table 1. 
Table 1 Shear testing of DeGrussa Mine paste backfill

\begin{tabular}{|c|c|c|c|c|}
\hline Sample & $\begin{array}{c}\text { Mixing } \\
\text { time (s) }\end{array}$ & $\begin{array}{c}\text { Rotary } \\
\text { viscometry yield } \\
\text { stress }(\mathrm{Pa})\end{array}$ & $\begin{array}{c}100 \mathrm{~mm} \\
\text { cylinder } \\
\text { slump (mm) }\end{array}$ & $\begin{array}{c}100 \mathrm{~mm} \\
\text { cylinder yield } \\
\text { stress (Pa) }\end{array}$ \\
\hline Surface & 0 & 248 & 25 & 429 \\
\hline Surface & 30 & 205 & 35 & 327 \\
\hline Surface & 60 & 177 & 39 & 291 \\
\hline Surface & 90 & 168 & 50 & 209 \\
\hline Surface & 120 & 160 & 55 & 178 \\
\hline Surface & 150 & 155 & 61 & 143 \\
\hline $\begin{array}{l}\text { Underground } \\
\text { measurements }\end{array}$ & & 95 & 68 & 107 \\
\hline
\end{tabular}

It is clear that further mixing of the paste backfill results in increased fluidity, however a further 2.5 minutes of mixing still did not meet the degree of fluidity present after the paste backfill had passed through the entire reticulation system from surface to the stope to be filled.

Figure 7 is a graphic representation of the slump and yield stress data presented in Table 1 . The blue, red and green arrows indicate the slump height and viscometer and slump yield stress value measurements of the paste backfill delivered at the stope respectively.

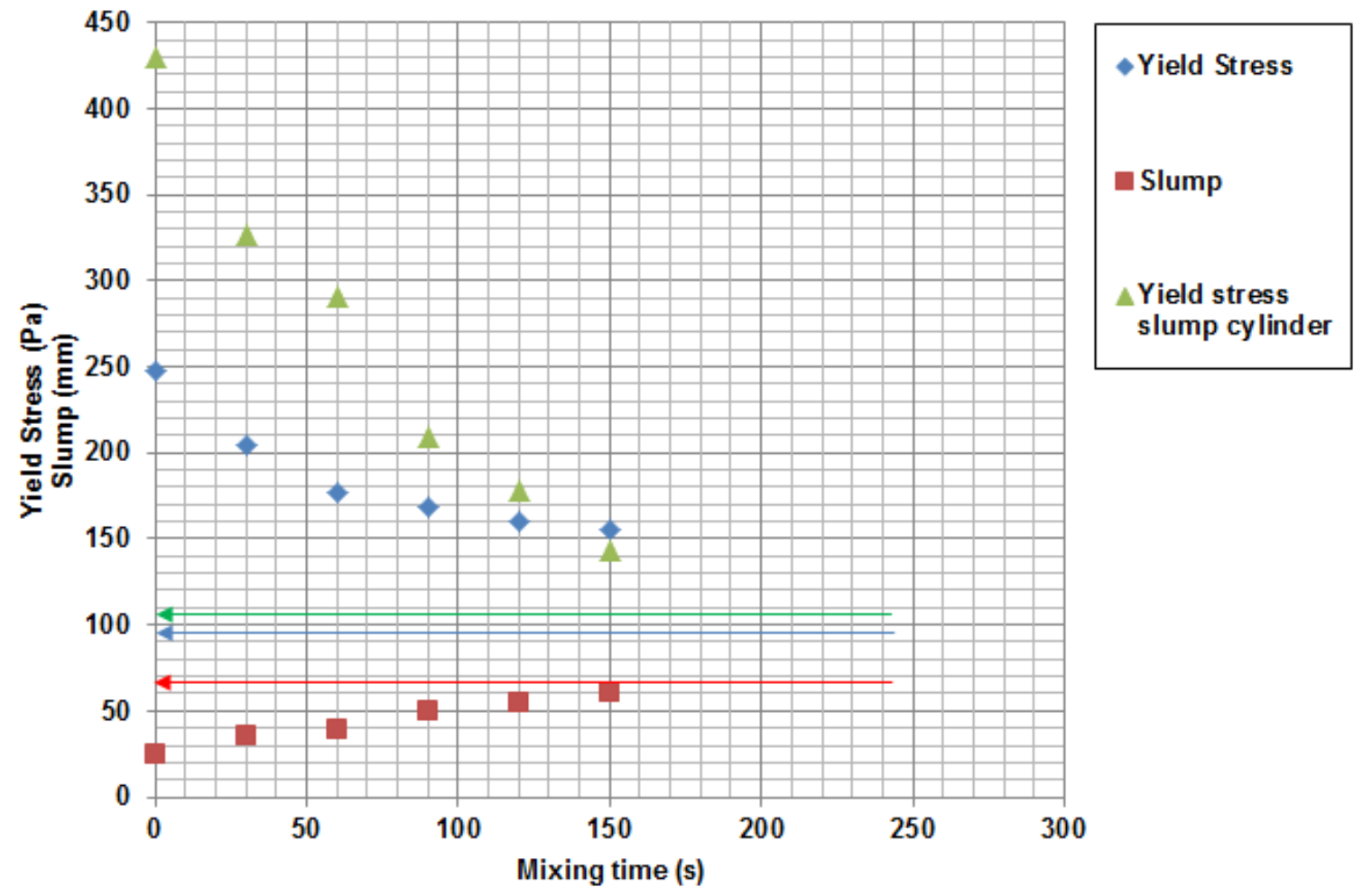

Figure 7 Graph of slump and viscosity versus mixing time 
The WTC has carried out pipe loop test work on a cemented DeGrussa Mine paste product at 78 wt\% solids similar to the current DeGrussa Mine paste backfill. The yield stress determined through the pipeline test work for the fully sheared homogeneous DeGrussa Mine paste backfill at $78 \mathrm{wt} \%$ solids was approximately $110 \mathrm{~Pa}$.

If the paste reticulation pipeline is sized on either the slump or rheometer viscosity test work the yield stress would be between 150 and $290 \%$ greater than the actual $110 \mathrm{~Pa}$. This exaggerated yield stress would result in the selection of a larger than necessary pipeline than if the paste was fully sheared. Furthermore, the 'unsheared' paste yield stress suggests that the paste backfill would not be able to flow through the longer reticulation routes without using additional head provided by a pump.

It is further interesting to note that in all the relationships shown in Figure 7, a limiting factor, or a constant rheology does not appear to have been achieved, although all the relationships appear to be tending towards a limiting, or constant, value.

\subsection{Paste manufacture solution}

In order to achieve sufficient mixing of the paste backfill it was proposed by the WTC that a Warman ${ }^{\circledR}$ AHF centrifugal pump be installed which would accept the total paste mixer discharge and then recirculate it back into the mixer discharge hopper prior to entering the paste backfill reticulation system.

The objective was to operate the pump as a high shear mixer imparting a high degree of energy to the paste. To this end a $110 \mathrm{~kW}, 4$ pole WEG Mining motor was fitted to a Warman ${ }^{\circledR}$ 3DD AHF all metal (A05) centrifugal froth pump with a packed or mechanical gland to prevent gland water dilution of the paste backfill, Figure 8.

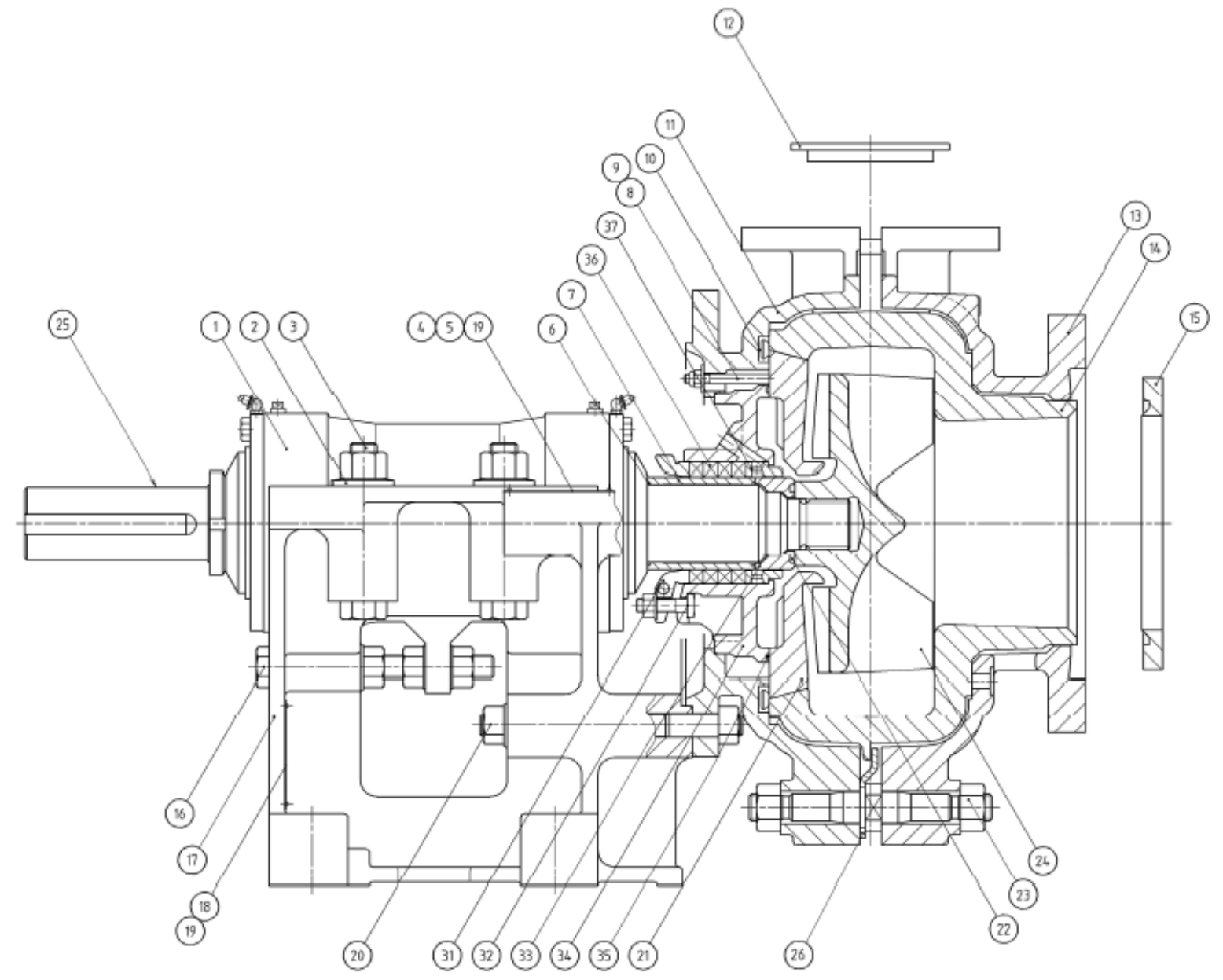

Figure 8 Warman ${ }^{\circledR}$ 3DD AHF centrifugal froth pump general arrangement (after Warman International Limited 1998) 
The pump specified was a froth pump to include both a larger suction inlet, to cater for the viscous paste backfill from the paste mixer, as well as the open scoop impellor design to force the viscous paste into the impellor.

The in situ pump installation is shown in Figure 9 and the paste pumping configuration in Figure 10.
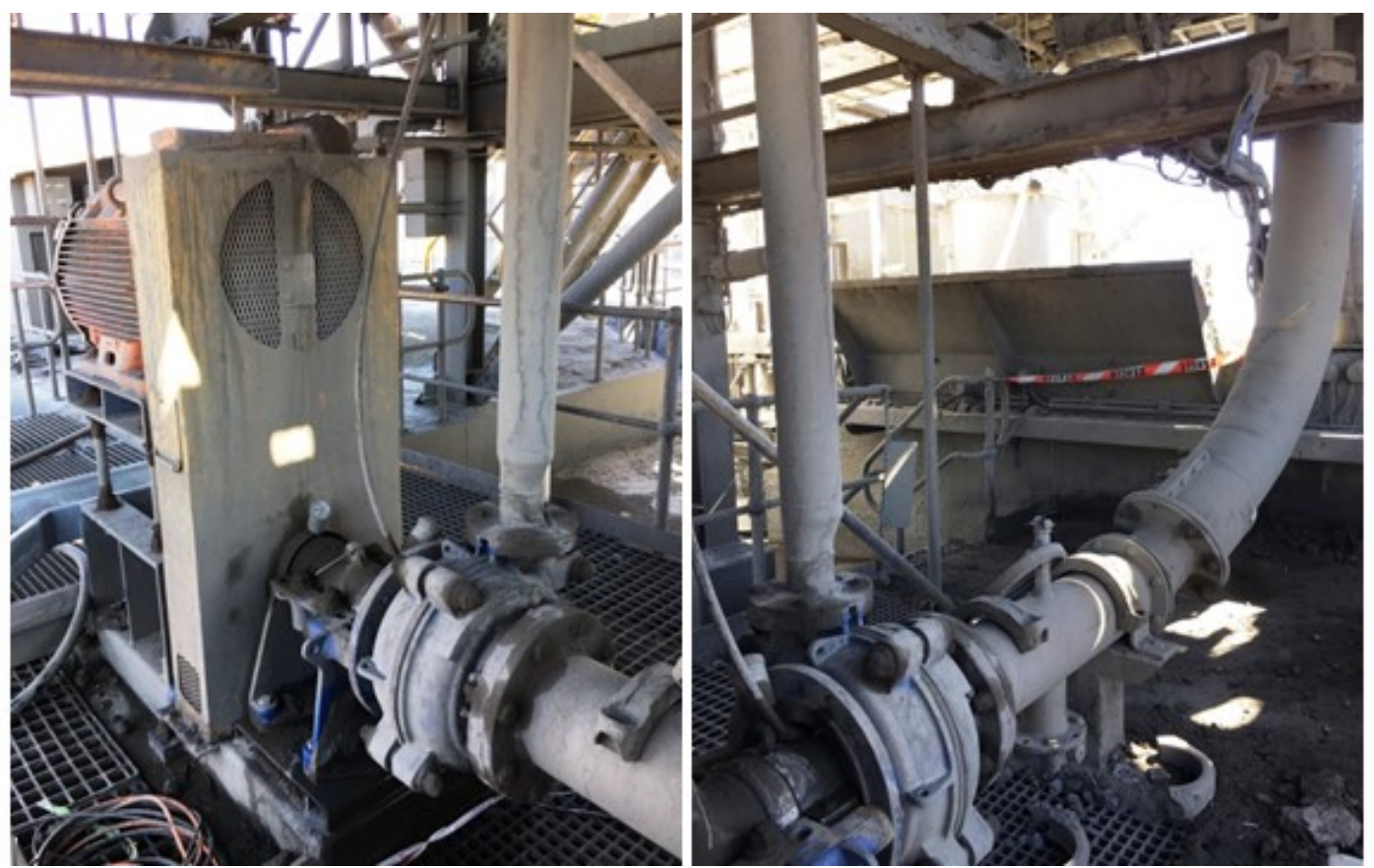

Figure 9 Warman ${ }^{\circledR}$ 3DD AHF centrifugal froth pump in situ installation

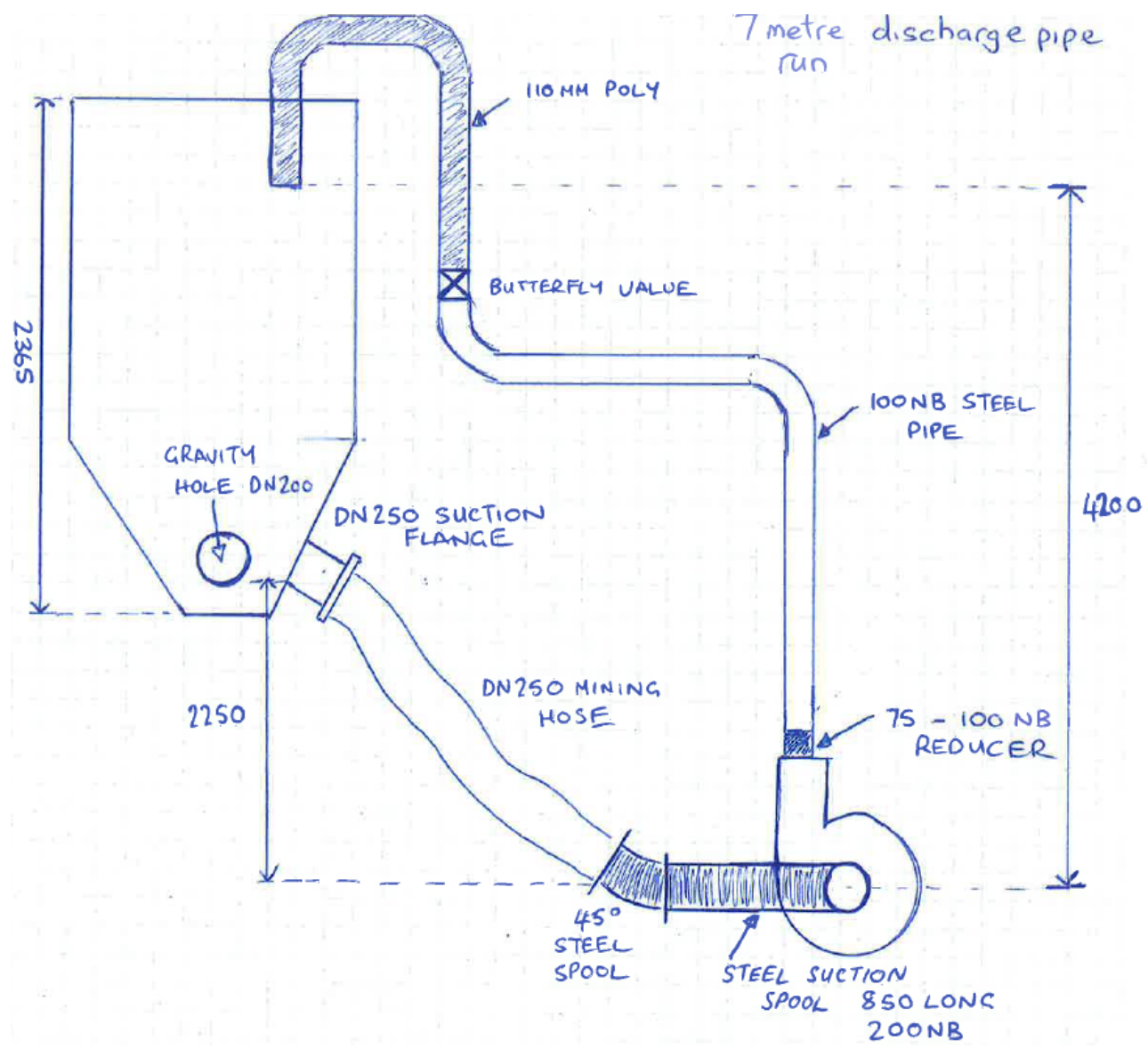

Figure 10 Warman ${ }^{\circledR}$ 3DD AHF centrifugal froth pump paste conditioning circuit 
DeGrussa Mine then carried out tests where the paste mixer product was fed through the froth pump and the yield stress compared to the paste mixer product. These results are given in Figure 11.

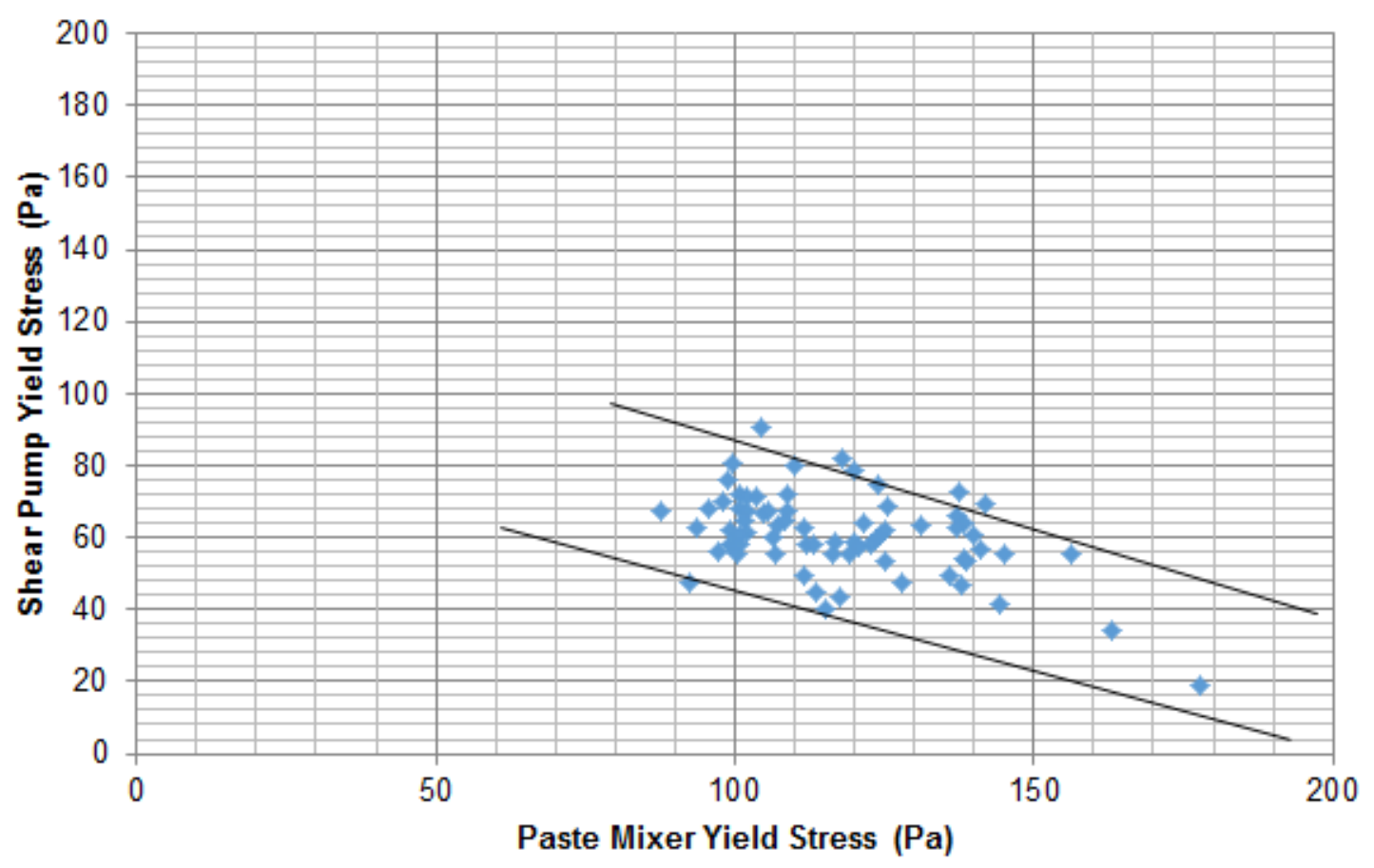

Figure 11 Warman ${ }^{\circledR}$ 3DD AHF shear pump yield stress versus paste mixer yield stress

The results in Figure 11 have been normalised for a 78 wt\% solids. There is still a wide variation in results probably pertaining to measurement variations as well as the full paste mixer output not being passed through the pump. In general, these normalised results indicate a $48 \%$ decrease in the yield stress on a single pass through the Warman ${ }^{\circledR}$ 3DD AHF centrifugal froth pump.

This clearly shows that insufficient mixing and shear takes place in the paste mixer resulting in inconsistently mixed paste, affecting reticulation. The simple installation of an all metal Warman ${ }^{\circledR}$ froth pump can remediate the situation without resulting in reduced flow rates that would be experienced with increased mixer retention times.

\section{$5 \quad$ Outcome}

Figure 12 shows the operating pressure gradients, red data points, which have been recorded on DeGrussa Mine prior to the installation of the paste backfill froth pump for shear conditioning. It will be noted that there is a broad range of measured pressure gradients, probably owing to paste backfill variability and system operating methodology. The average pressure gradient of this total dataset is $7.85 \mathrm{~Pa}$ with a standard deviation of 1.76 .

Design work carried out by the WTC for DeGrussa Mine on designing the reticulation requirements for various stopes has successfully used an average pressure gradient of approximately $8 \mathrm{kPa} / \mathrm{m}$ for a flow of $80 \mathrm{~m}^{3} / \mathrm{hr}$.

The blue data in Figure 12 are the pressure gradients recorded underground while the froth pump carrying out the shear conditioning is in operation. The average pressure gradient for this data is $5.48 \mathrm{~Pa}$ with a standard deviation of 1.46 .

This is a decrease of $30 \%$ in the pipeline pressure gradient which is manifest as an improvement in the flow rate or distance travelled through the pipeline, i.e. the paste backfill can be transported $30 \%$ farther in the fully sheared condition than the existing condition delivered from the paste mixer. 


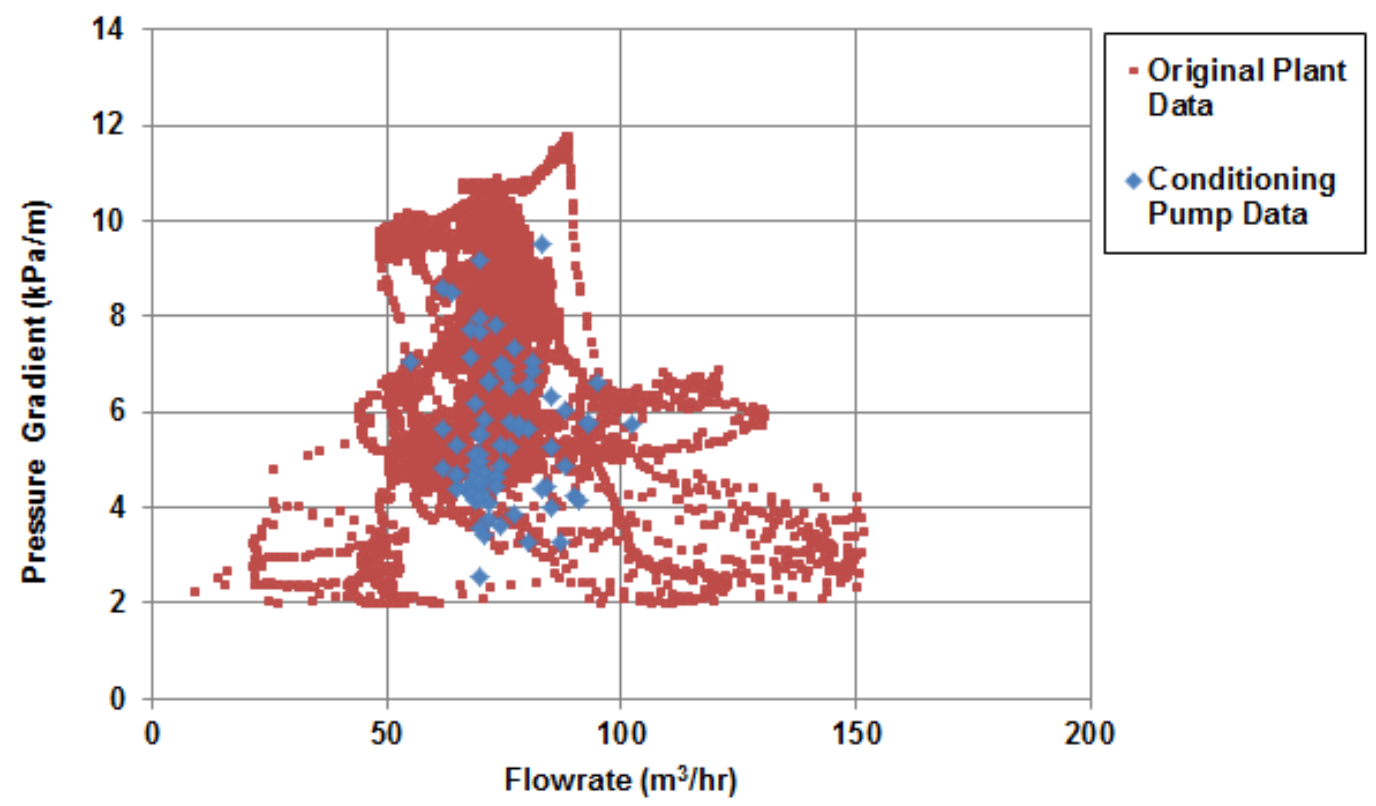

Figure 12 Flow rate versus pressure gradient for a specific time period less outliers

Of interest is that the average flow rate for both sets of data is approximately $74 \mathrm{~m}^{3} / \mathrm{hr}$; however, the variation inflow rate for the original data is greater than for the froth pump data.

\section{Conclusions}

The outcome of installing the Weir Minerals Warman ${ }^{\circledR}$ 3DD AHF all metal centrifugal pump to deliver further shear to the cemented paste backfill results in several benefits to DeGrussa Mine:

- Better quality paste backfill in respect to a better mixed final product, resulting in improved homogeneity of the tailings, water and binder.

- Consistent pressure gradients throughout the entire pipeline length enabling more precise reticulation design.

- More stable pipeline operation enabling greater understanding of the paste behaviour and the ability to anticipate anomalous behaviour.

- Reduced likelihood of pipe wear owing to freefall.

- The ability to deliver paste backfill to the farthest reaches of the mining operation.

\section{Acknowledgements}

The authors would like to acknowledge the staff and management of DeGrussa Mine for the support and commitment in delivering the in situ work and installations required to develop the paste backfill solutions on DeGrussa Mine; as well as the staff and management of Sandfire Resources for their support and permission to publish this work.

\section{References}

Clark, IH 1988, 'The properties of hydraulically placed backfill', Backfill in South African Mines, The Southern African Institute of Mining and Metallurgy, Johannesburg, pp. 15-33.

Pashias, N, Boger, DV, Summers, J \& Glenister, DJ 1996, 'A fifty cent rheometer for yield stress measurement', Journal of Rheology, vol. 40 , p. 6.

Warman International Limited 1998, 3 Frame D, Type AHF metal lined components diagram, drawing number: A1-110-0-135161Rev1. 
